\title{
THE TAKAGA OPERATOR, BERNOULLI SEQUENCES, SMOOTHNESS CONDITIONS, AND FRACTAL CURVES
}

\author{
ANCA DELIU AND PETER WINGREN
}

(Communicated by Palle E. T. Jorgensen)

\begin{abstract}
We map Lipschitz spaces and functions of bounded variation by the operator $\sigma: \varphi(x) \rightarrow \sum_{0}^{\infty} 2^{-n} \varphi\left(2^{n} x\right), x \in[0,1]$, and we estimate the Hausdorff measure of $\sigma(\varphi)$. We furthermore introduce a class of continuous and nowhere differentiable functions on $[0,1]$ which we call $\mathscr{T}$. We make a refined analysis of the fractal and smoothness properties of the functions in $\mathscr{I}$ and study the relationship between the two. We show that all the functions in $\mathscr{T}$ have box dimension equal to $\frac{1}{2}$, with respect to the dimension family $\left\{t /\left(\log \frac{1}{t}\right)^{s}: s \in \mathbb{R}^{+}\right\}$, but that their order of smoothness covers a wide range.
\end{abstract}

\section{INTRODUCTION}

Functions of the type

$$
\sigma_{a, b}(\varphi)(x)=\sum_{0}^{\infty} a^{-n} \varphi\left(b^{n} x\right), \quad x \in \mathbb{R},
$$

have been used, for different values of $a$ and $b$ and special choices of $\varphi$, as examples of continuous and nowhere differentiable functions, for instance by Cellerier [HOB, p. 402], Weierstrass [WEI], Takaga [TAK], and van der Waerden [WAE]. Moreover the Hausdorff measure and fractal properties of the graphs of the functions (1.1) have been considerably studied, for instance by Besicovitch and Ursell (see [FAL1, p. 115]), Mandelbrot [MAN, p. 328], Mauldin and Williams [MAL], and several others.

In this paper we discuss some relations between smoothness and fractal properties for special classes of functions. These classes are defined as images, under the mapping $\sigma=\sigma_{2,2}$, called in this paper, the Takaga operator, of (i) certain Lipschitz spaces, (ii) the space of continuous, bounded functions, of uniform bounded variation on the intervals determined by the integers, and (iii) a class of sawteeth functions, created by all but a countable number of Bernoulli sequences. Each of these classes contains Takaga's nowhere differentiable function, $T(t):=\sigma(\inf \{|t-y|: y \in \mathbb{Z}\})$. The function $T(t)$ was first constructed

Received by the editors March 11, 1992 and, in revised form, October 24, 1992.

1991 Mathematics Subject Classification. Primary 28A78; Secondary 42A32.

Key words and phrases. Nowhere differentiable, Hausdorff dimension.

The first author was partially supported by NSF Grant DMS 9991381.

The second author was partially supported by the Swedish Science Research Council, the Royal Academy of Sciences, and Magnusson's Foundation. 
by Takaga in 1903 [TAK] (see also the next section) as a simpler example than Weierstrass's from 1873 [WEI], of a nowhere differentiable continuous function. The function has then been rediscovered many times, for instance by van der Waerden [WAE], who gave a construction very similar to Takaga's. The construction of van der Waerden was later improved by de Rham [RHA] to a function identical to Takaga's.

To be able to describe our results we need some preparations. The first of these is to give some definitions of function spaces (see, for instance, [BIN, JWL, STE, WIN]. If $f \in \operatorname{Lip}(\omega(|h|)), \mathbb{R})$, where $\omega$ is a modulus of smoothness, then, by definition, $f$ is bounded and there exist constants $c_{1}>0$ and $c_{2}>0$ so that the first difference $\Delta_{h} f(x)=f(x+h)-f(x)$ satisfies

$$
\left|\Delta_{h} f\right|<c_{1} \omega(|h|),
$$

for every $|h|<c_{2}$. If $\omega(|h|)=|h|^{\alpha}, 0<\alpha \leq 1$, then we get the usual Lipschitz spaces $\operatorname{Lip}(\alpha, \mathbb{R})$ of functions which satisfy a Hölder condition of order $\alpha$, and if the first difference is replaced by the second difference and $\alpha=1$ then we get the Zygmund class $\Lambda^{1}(\mathbb{R})$. The restriction to $[0,1]$ of, for example, $\operatorname{Lip}(\alpha, \mathbb{R})$ is denoted by $\operatorname{Lip}(\alpha,[0,1])$. To make notation simpler we will not always make a distinction between a function and its graph.

The second preparation is to give a motivation for introducing an additional dimension family.

The following embeddings hold for every $0<\alpha<1$ :

$$
\operatorname{Lip}(1) \subset \Lambda^{1} \subset \operatorname{Lip}\left(|h| \log \frac{1}{|h|}\right) \subset \operatorname{Lip}(\alpha) .
$$

The inclusions (1.2) together with a well-known result of Besicovitch (see [FAL1, p. 113]) that

$$
f \in \operatorname{Lip}(\alpha) \Rightarrow \operatorname{dim}_{H}(\operatorname{graph} f) \leq 2-\alpha,
$$

give that every function in $\operatorname{Lip}\left(|h| \log \frac{1}{|h|}\right)$ has Hausdorff dimension 1 (as a matter of fact every function in $\bigcap_{\alpha<1} \operatorname{Lip}(\alpha)$ has Hausdorff dimension 1$)$. However Hata [HAT] has pointed out that it is easy to see that Takaga's function $T(t)$ belongs to $\operatorname{Lip}\left(|h| \log \frac{1}{|h|}\right)$ (which also follows from our theorems), and this means that $\operatorname{Lip}\left(|h| \log \frac{1}{|h|}\right)$ contains both functions which are considered to be fractal and which are not, even if they all have dimension 1 . Therefore we add to the dimension family $\left\{t^{s}: s \in \mathbb{R}^{+}\right\}$the family $\left\{t /\left(\log \frac{1}{t}\right)^{s}: s \in \mathbb{R}^{+}\right\}$. This allows us to make a finer dimension analysis (see [FAL2] for a discussion) and to some extent to separate functions with fractal behaviour from others.

The third, and last, preparation is to give the notation associated with the added dimension family. The easiest way is to add "with respect to $\left\{t /\left(\log \frac{1}{t}\right)^{s}: s\right.$ $\left.\in \mathbb{R}^{+}\right\}$" to the usual notation. For instance we will say Hausdorff dimension with respect to $\left\{t /\left(\log \frac{1}{t}\right)^{s}: s \in \mathbb{R}^{+}\right\}$(which we temporarily also name logarithmic Hausdorff dimension), and box dimension with respect to $\left\{t /\left(\log \frac{1}{t}\right)^{s}: s \in \mathbb{R}^{+}\right\}$. We also add a little more notation. Recall that the Hausdorff measure of a set with respect to $h_{s}(t)=t /\left(\log \frac{1}{t}\right)^{s}$ is defined by

$$
\lim _{\varepsilon \rightarrow 0} \inf _{\left\{U_{i}\right\}} \sum h_{s}\left(\left|U_{i}\right|\right)
$$


where $\left\{U_{i}\right\}$ is a countable covering of the measured set, $\left|U_{i}\right|$ denotes the diameter of the set $U_{i}$, and $\left|U_{i}\right| \leq \varepsilon$. If we just consider the sum $\sum_{\left\{U_{i}\right\}} h_{s}\left(\left|U_{i}\right|\right)$ for dyadic cubes of equal sidelength (which is the case in this paper), then we name the sum above box sum with respect to $h_{s}(t)$, and the possible limits of (1.4) lower $\left(\underline{\mathrm{im}}^{B}\right)$ and upper $\left(\overline{\lim }^{B}\right)$ box sum limits, and if they coincide, just the box sum limit $\left(\lim ^{B}\right)$ with respect to $\left\{h_{s}(t): s \in \mathbb{R}^{+}\right\}$, respectively. In what follows we will use $\left|Q_{i}\right|$ as notation for the sidelength of $Q_{i}$. It is easy to see that this does not affect the existence and relation between the limits above. Observe also that positive (and finite) lower and upper box sum limits with respect to $h_{s}(t)=t /\left(\log \frac{1}{t}\right)^{s}, s=s_{0}$, is a sufficient condition for the box dimension with respect to $\left\{t /\left(\log \frac{1}{t}\right)^{s}: s \in \mathbb{R}^{+}\right\}$to exist, and be equal to $s_{0}$.

We are now prepared to describe our results which are of three types: (I), (II), (III).

(I) We generalize (Theorem 2.1) the well-known result of Besicovitch for Lipschitz spaces (1.3), and we also show (Theorem 2.3) how the local behaviour of $f$ is inherited by $\sigma(f)$. These two theorems together can be used to estimate the Hausdorff measure of graph $(\sigma(f))$.

(II) We then use a global condition on $f$, given in terms of bounded variation, and find an estimate for the Hausdorff measure of $\sigma(f)$ with respect to $t / \log \frac{1}{t}$ (Theorem 2.4). Already the theorems of types (I) and (II) improve the result for the Takaga function in [MAL], $\sigma$-finite $\left(t / \log \frac{1}{t}\right)$-Hausdorff measure, to finite $\left(t / \log \frac{1}{t}\right)$-Hausdorff measure. Further improvement is made by the theorems in (III), to finite $\left(t /\left(\log \frac{1}{t}\right)^{1 / 2}\right)$-Hausdorff measure, but a description of those theorems needs a few more definitions.

(III) We establish a one-to-one mapping of $(0,1]$ onto a class of nowhere differentiable functions. Let $\varepsilon_{k}(a)$ be defined by $\varepsilon_{k}(a)=2 a_{k}-1$, where $a=$ $0 . a_{1} a_{2} a_{3} \ldots$ is the binary expansion of $a$, where uniqueness has been achieved by excluding expansions which end with an infinite number of zeros; in other words $\left\{a_{i}\right\}_{1}^{\infty}$ are all Bernoulli sequences except those with a finite number of 1 's. Let then

$$
\begin{aligned}
& \operatorname{Int}(x):=\text { the integer part of the real number } x, \\
& \operatorname{dist}(x):=\inf \{|y-x|: y \in \mathbb{Z}\}, \\
& \gamma_{a}(x):=\varepsilon_{\operatorname{Int}}(x)+1(a) \times \operatorname{dist}(x), x>0, \\
& \Gamma:=\left\{\gamma_{a}: a \in(0,1]\right\}, \\
& \mathscr{T}:=\sigma(\Gamma) .
\end{aligned}
$$

Then we prove the following properties for the class $\mathscr{T}$.

(1) $\mathscr{T}$ consists of nowhere differentiable functions (Theorem 2.9).

(2) A refined dimension investigation shows that the box dimension with respect to $\left\{t /\left(\log \frac{1}{t}\right)^{s}: s \in \mathbb{R}^{+}\right\}$exists, and moreover it has the same value, $\frac{1}{2}$, for every function in $\mathscr{T}$.

(3) As a consequence of (2), for every $a \in(0,1]$, the Hausdorff measure of the graph of $\tau_{a}=\sigma\left(\gamma_{a}\right)$ is finite with respect to $t /\left(\log \frac{1}{t}\right)^{1 / 2}$ (Theorem 2.6). Notice that the upper bound of the logarithmic Hausdorff dimension of the functions in $\mathscr{T}$, provided by Theorem 2.6 , cannot be deduced analogously to Besicovitch's result (1.3) from Theorem 2.1, since, for instance, $T(t) \notin$ $\operatorname{Lip}\left(|h|\left(\log \frac{1}{\mid h}\right)^{1 / 2}\right)$.

(4) The class $\mathscr{T}$ contains functions which have very different smoothness 
properties. We will show that $\mathscr{T}$ contains both functions with second difference $O\left(|h| \log \frac{1}{|h|}\right)$ best possible, and functions with second difference $O(|h|)$.

The results in (2) and (4) together are interesting and lead us to conjecture that $\mathscr{T}$ contains functions for which the Hausdorff dimension and box dimension with respect to the dimension family $\left\{t /\left(\log \frac{1}{t}\right)^{s}: s \in \mathbb{R}^{+}\right\}$are distinct, even though we look upon this conjecture more as a suggested direction of work, than an absolute prediction. Compare this with the well-known conjecture that the Hausdorff dimension and box dimension of the Weierstrass function are the same.

\section{THEOREMS AND PROOFS}

Theorem 2.1. If $\omega$ is a modulus of smoothness, $\lim _{|h| \rightarrow 0}(|h| / \omega(|h|))=0$, and $f \in \operatorname{Lip}(\omega(|h|),[0,1])$, then the Hausdorff measure of $\operatorname{graph}(f)$ is finite with respect to $t^{2} / \omega(t)$.

Proof. Let $[x, x+\delta]$ be a $\delta$-interval on $[0,1]$ and $\|f\|$ the norm of $f$ in $\operatorname{Lip}(\omega(|h|),[0,1])$. Thus

$$
|f(x+h)-f(x)| \leq\|f\| \omega(|h|), \quad x \in[0,1],
$$

and it follows that the restriction of $f$ to $[x, x+\delta]$ may be covered by $M$ squares of sidelength $\delta$, where

$$
M=\operatorname{Int} \frac{\|f\| \omega(\delta)}{\delta}+2 .
$$

Hence the whole graph of $f$ may be covered by $N$ dyadic $\delta$-squares $Q_{1}$, $Q_{2}, \ldots, Q_{N}$, where $N=M \cdot 1 / \delta$, and we have the estimation

$$
\inf _{|Q|=\delta} \sum_{Q \cap F}|Q|^{2} / \omega(|Q|) \leq\|f\|+2 \delta / \omega(\delta) .
$$

Now let $\delta$ approach zero and we get

$$
\varlimsup^{B}(f)<+\infty
$$

with respect to the function $t^{2} / \omega(t)$, which proves the theorem.

Remark 2.2. Application of Theorem 2.1, for $\omega(\delta)=\delta^{\alpha}, 0<\alpha<1$, gives Besicovitch's result 1.3. The Takaga function belongs to $\operatorname{Lip}\left(|h| \log \frac{1}{|h|},[0,1]\right)$, and hence Theorem 2.1 improves the result in [MAL, p. 799] from $\sigma$-finiteness to finiteness with respect to $t / \log \frac{1}{t}$.

This result will be further improved in Theorem 2.6. The next theorem may be generalized considerably, but then we would have to impose less simple conditions on the modulus of smoothness, and we will avoid that in this paper.

Theorem 2.3. Let $Z$ be the operator which maps a bounded function $f \in C^{0}(\mathbb{R})$ to $\frac{1}{2} f(2 x)$ and let $\sigma$ be the operator defined by $\sigma(f)=\sum_{n=0}^{\infty} Z^{n}(f)$. Then

(i) $\operatorname{Lip}(\alpha, \mathbb{R})$ is invariant under the operator $\sigma$ for every $\alpha<1$,

(ii) $\operatorname{Lip}(1, \mathbb{R})$ is not invariant under $\sigma$, and

(iii) $\operatorname{Lip}\left(|h|\left(\log \frac{1}{|h|}\right)^{m}, \mathbb{R}\right)$ is mapped into $\operatorname{Lip}\left(|h|\left(\log \frac{1}{|h|}\right)^{m+1}\right)$ by $\sigma$, for every nonnegative integer $m$. 
Proof. Let $0<\alpha<1$ and let $\|f\|$ be the norm of $f$ in $\operatorname{Lip}(\alpha, \mathbb{R})$. Part (i) is proved if we show that there exists a positive constant $c$ so that $\sigma f(x)$ is bounded by $c$ and

$$
|\sigma f(x+h)-\sigma f(x)| \leq c|h|^{\alpha} .
$$

It is easy to see that, by the following estimates, inequality $(2.5)$ is true for $c=\|f\| /\left(1-2^{\alpha-1}\right)$ :

$$
\begin{aligned}
\left|\Delta_{h} \sigma f(x)\right| & =\sum_{0}^{\infty} 2^{-n}\left|f\left(2^{n} x+2^{n} h\right)-f\left(2^{n} x\right)\right| \\
& \leq\|f\| \sum_{0}^{\infty} 2^{-n}\left(2^{n}|h|\right)^{\alpha}=\frac{\|f\|}{1-2^{\alpha-1}}|h|^{\alpha} .
\end{aligned}
$$

Part (ii) is well known since the Takaga function provides a counterexample. Part (iii) is proved if we show that to a function $g \in \operatorname{Lip}\left(|h|\left(\log \frac{1}{|h|}\right)^{m}, \mathbb{R}\right)$ there exists a $c>0$ so that $\sigma g(x)$ is bounded by $c$ and

$$
|\sigma g(x+h)-\sigma g(x)| \leq c|h|\left(\log \frac{1}{|h|}\right)^{m+1},
$$

for all $|h|$ less than a positive constant $c_{m}$. The constant $c_{m}$ is chosen so that $h\left(\log \frac{1}{h}\right)^{m}$ is increasing in the interval $\left(0, c_{m}\right)$. Since $g$ is bounded then $\sigma g$ is bounded by construction and it remains to prove (2.6). We have

$$
|\sigma g(x+h)-\sigma g(x)| \leq \sum_{0}^{\infty} 2^{-n}\left|g\left(2^{n}(x+h)\right)-g\left(2^{n} x\right)\right|
$$

Let $\|g\|$ be the norm of $g$ in $\operatorname{Lip}\left(|h|\left(\log \frac{1}{|h|}\right)^{m}\right)$. We denote by $S_{\infty}$ the righthand side of (2.7) and by $S_{N}$ the corresponding $N$ th partial sum, where $N$ is the unique nonnegative number such that $2^{-(N+1)} \leq|h|<2^{-N}$. Then

$$
S_{N} \leq \sum_{0}^{N} 2^{-n}\|g\| 2^{n} h\left|\left(\log \frac{1}{2^{n}|h|}\right)^{m}=\|g\| \sum_{0}^{N}\right| h \mid\left(\log \frac{1}{2^{n}|h|}\right)^{m}
$$

and since $|h|\left(\log \frac{1}{2^{n}|h|}\right)^{m}$ is increasing on the interval $\left[2^{-N-1}, 2^{-N}\right)$,

$$
\begin{aligned}
S_{N} & \leq\|g\| \sum_{0}^{N} 2^{-N}\left(\log \frac{1}{2^{n} 2^{-N}}\right)^{m} \leq(\log 2)^{m} \times\|g\| 2^{-N} N^{m} \sum_{0}^{N}\left(\frac{N-n}{N}\right)^{m} \\
& \leq(\log 2)^{m} \times\|g\| 2^{-N} N^{m}(N+1) 2^{-1} \leq C\|g\||h|\left(\log \frac{1}{|h|}\right)^{m+1},
\end{aligned}
$$

for some $C>0$. It remains to estimate $S_{\infty}-S_{N}$ but that is easy, since $g$ is bounded by $\|g\|$, and

$$
S_{\infty}-S_{N}=\sum_{N+1}^{\infty} 2^{-n}\left|g\left(2^{n}(x+h)\right)-g\left(2^{n} x\right)\right| \leq 2^{-(N+1)} 4\|g\| \leq|h| 4\|g\| .
$$

Adding up gives

$$
|\sigma g(x+h)-\sigma g(x)| \leq C|h|\left(\log \frac{1}{|h|}\right)^{m+1},
$$

for some $C>0$, and the proof is complete. 
Theorem 2.4. Let $f$ be a continuous, bounded function, and suppose that $f$ has uniform bounded variation on $\{[k, k+1]: k \in \mathbb{Z}\}$. Then $\sigma(f)$ (restricted to $[0,1])$ has finite upper box sum limit (and hence finite Hausdorff measure) with respect to $h(t)=t / \log \frac{1}{t}$.

Proof. Let $v=2 \sup _{x \in \mathbf{R}}|f(x)|$ be twice the $L_{\infty}$ norm of $f$ on $\mathbb{R}, V_{k}$ the total variation of $f$ on $[k, k+1], k \in \mathbb{Z}$, and $V=\sup _{k}\left\{V_{k}: k \in \mathbb{Z}\right\}$. Let

$$
f_{\nu}(t)=\sum_{i=0}^{\nu} 2^{-i} f\left(2^{i} t\right)
$$

Then

$$
\left|\sigma(f)-f_{\nu}\right| \leq\left|\sum_{i=\nu+1}^{\infty} 2^{-i} f\left(2^{i} t\right)\right| \leq v 2^{-\nu} .
$$

Hence $\mathscr{N}\left(f, 2^{-\nu}\right)$, the number of dyadic cubes of sidelength $2^{-\nu}$ intersecting $\operatorname{graph}(f)$, is bounded by

$$
\mathscr{N}\left(\sigma(f), 2^{-\nu}\right) \leq \mathcal{N}\left(f_{\nu}, 2^{-\nu}\right)+2^{\nu} \times 2 \times \frac{v 2^{-\nu}}{2^{-\nu}}
$$

For any continuous function $\psi$ on $[0,1]$ let

$$
\operatorname{Var}_{\nu}(\psi)=\sum_{k=0}^{2^{\nu}-1} \sup _{I_{\nu k}} \psi-\inf _{I_{\nu k}} \psi
$$

where $I_{\nu k}=\left[2^{-\nu} k, 2^{-\nu}(k+1)\right]$. Then

$$
\operatorname{Var}_{\nu}\left(f_{\nu}\right) \leq \sum_{i=0}^{\nu} \operatorname{Var}_{\nu} 2^{-i} f\left(2^{i} t\right) \leq(\nu+1) V
$$

Hence

$$
\mathscr{N}\left(f_{\nu}, 2^{-\nu}\right) \leq \frac{(\nu+1) V}{2^{-\nu}}+2^{\nu} \times 2
$$

Finally

$$
\begin{aligned}
& \underset{\nu \rightarrow \infty}{\limsup } \mathcal{N}\left(\sigma(f), 2^{-\nu}\right) h\left(2^{-\nu}\right) \\
& \quad \leq \limsup _{\nu \rightarrow \infty}\left(\frac{(\nu+1) V}{2^{-\nu}}+2^{\nu} \times 2+2^{\nu} \times 2 v\right) h\left(2^{-\nu}\right) \leq V .
\end{aligned}
$$

We will need the following combinatorial identity (see [HAG]) in the proof of our next theorem.

Lemma 2.5. If $m$ is a positive integer then

$$
\sum_{k=0}^{m} k\left(\begin{array}{c}
2 m \\
k
\end{array}\right)=m \cdot 2^{2 m-1}
$$

Proof. From

$$
\sum_{k=1}^{m} k\left(\begin{array}{c}
2 m \\
k
\end{array}\right)=\sum_{k=1}^{m}(2 m-k+1)\left(\begin{array}{c}
2 m \\
k-1
\end{array}\right)=\sum_{k=0}^{m-1}(2 m-k)\left(\begin{array}{c}
2 m \\
k
\end{array}\right)
$$


follows

$$
2 \sum_{k=1}^{m} k\left(\begin{array}{c}
2 m \\
k
\end{array}\right)=m\left[2 \sum_{k=0}^{m-1}\left(\begin{array}{c}
2 m \\
k
\end{array}\right)+\left(\begin{array}{c}
2 m \\
m
\end{array}\right)\right]=m \cdot 2^{2 m}
$$

and thus

$$
\sum_{k=1}^{m} k\left(\begin{array}{c}
2 m \\
k
\end{array}\right)=m \cdot 2^{2 m-1}
$$

Theorem 2.6. The box dimension of graph $\tau, \tau \in \mathscr{T}$, with respect to $\left\{t /\left(\log \frac{1}{t}\right)^{s}\right.$ : $\left.s \in \mathbb{R}^{+}\right\}$exists and is $s=\frac{1}{2}$ and thus the Hausdorff measure with respect to $t /\left(\log \frac{1}{t}\right)^{1 / 2}$ is finite.

Proof. The proof will be divided in three steps: Let $\tau_{n}$ denote the $n$th partial sum in the definition of $\tau=\sigma\left(\gamma_{a}\right)$.

(I) It is easy to see that:

(i) The nodes of the continuous and piecewise linear $\tau_{0}(t)=\gamma_{a}(t)$ are at $t$-distance $\frac{1}{2}$ or 1 , for all $a \in[0,1)$.

(ii) If $2^{-i} \gamma_{a}\left(2^{i} t\right)$ has a node at $t_{0}$, then all $2^{-k} \gamma_{a}\left(2^{k} t\right), k>i$, have the value 0 at $t_{0}$. It follows from (ii) that

(iii) if $\tau_{i}(t)$ has a node at $t_{0}$, then $\tau_{i}\left(t_{0}\right)=\tau_{k}\left(t_{0}\right), k \geq i$.

We may determine the variation of $\tau_{n}(t)$ on $[0,1]$ by adding, from left to right, the absolute slope value on each interval of length $2^{-n}$ and then multiplying by $2^{-n}$. It seems though difficult to reach a formula for $\operatorname{var}\left(\tau_{n}\right)$ in this way. The following observations lead us however immediately to the formula. First observe that if $\tau_{n}(t)$ has slope $l$ on the dyadic interval $I$, then by adding $2^{-(n+1)} \gamma\left(2^{n+1} t\right)$ we get $\tau_{n+1}(t)$ with slopes $l-1$ on one half of $I$ and $l+1$ on the other half of $I$. Hence if $\tau_{n}$ has $k_{1}$ intervals of slope $l$, then these intervals generate $k_{1}$ intervals of slope values $l-1$ and $l+1$, respectively. For adjacent slope values and their frequencies on $[0,1]$, we then have the situation in the following table:

\begin{tabular}{|l|ccccc|}
\hline Slope Values & $l-1$ & $l$ & $l+1$ & $l+2$ & $l+3$ \\
\hline Frequencies: & & & & & \\
for $\tau_{n}$ & 0 & $k_{1}$ & 0 & $k_{2}$ & 0 \\
for $\tau_{n+1}$ & $+k_{1}$ & 0 & $k_{1}+k_{2}$ & 0 & $k_{2}+$ \\
\hline
\end{tabular}

Hence the slopes of $\tau_{n}$ differ by multiples of 2 . We see from the table that a number in the second row and in column $l+1$ is the sum of the two numbers immediately above at the right and left in the first row. Observe secondly that the initial values are those in the following table:

\begin{tabular}{|c|ccc|}
\hline Slope Values & -1 & 0 & +1 \\
\hline Frequencies for $\tau_{0}:$ & 1 & 0 & 1 \\
\hline
\end{tabular}

Hence all the possible frequencies of absolute slopes values of the $n$th partial sum are given by the entries the $n$th row in Pascal's triangle. From this it 
follows that, for all $n \geq 1$,

$$
\operatorname{var}\left(\tau_{n-1}\right)=2^{-n} \sum_{k=0}^{n}|n-2 k|\left(\begin{array}{l}
n \\
k
\end{array}\right) .
$$

(II) We now turn to the estimation of the sum (2.8):

$$
\operatorname{var}\left(\tau_{2 m-1}\right)=2^{-2 m} \sum_{k=0}^{2 m}|2 m-2 k|\left(\begin{array}{c}
2 m \\
k
\end{array}\right)=2^{-2 m+2} \sum_{k=0}^{m}(m-k)\left(\begin{array}{c}
2 m \\
k
\end{array}\right) .
$$

By using the identity

$$
\sum_{k=0}^{m}\left(\begin{array}{c}
2 m \\
k
\end{array}\right)=2^{2 m-1}+\frac{1}{2}\left(\begin{array}{c}
2 m \\
m
\end{array}\right)
$$

Lemma 2.5, and the estimate of $\left(\begin{array}{c}2 m \\ m\end{array}\right)$ by Stirling's formula

$$
\left(\begin{array}{c}
2 m \\
m
\end{array}\right)=2^{2 m-1 / 2} m^{-1 / 2}\left(1+O\left(\frac{1}{m}\right)\right)
$$

we get

$$
\begin{aligned}
\operatorname{var}\left(\tau_{2 m-1}\right) & =2^{-2 m+2}\left(m \sum_{k=0}^{m}\left(\begin{array}{c}
2 m \\
k
\end{array}\right)-\sum_{k=0}^{m} k\left(\begin{array}{c}
2 m \\
k
\end{array}\right)\right) \\
& =2^{-2 m+2} \frac{m}{2}\left(\begin{array}{c}
2 m \\
m
\end{array}\right)=2 \sqrt{2 m-1}\left(1+O\left(\frac{1}{m}\right)\right) .
\end{aligned}
$$

Since $\operatorname{var}\left(\tau_{n}\right)$ is an increasing function of $n$ it follows that $\operatorname{var}\left(\tau_{n}\right)=$ $2 \sqrt{n}\left(1+O\left(\frac{1}{n}\right)\right)$.

(III) We will prove now that the graph of $\tau$ has a positive and finite box sum limit with respect to $t /\left(\log \frac{1}{t}\right)^{1 / 2}$, for abscissa values between 0 and 1 .

Let $\delta>0$. Cover the right half plane with the dyadic cubes of sidelength $2^{-n}, 2^{-n}<\delta$. The exact number of cubes which cover the nonconstant parts of $\tau_{n-1}$ is $\operatorname{var}\left(\tau_{n-1}\right) / 2^{-n}$. An upper bound for the number of cubes which cover the constant parts is $\frac{1}{2^{-n}}$. Furthermore we know from the construction that $\left|\tau(t)-\tau_{n-1}(t)\right|<2^{-n}$. This means that we just have to add $2 \times\left(1 / 2^{-n}\right)$ cubes to those covering $\operatorname{graph}\left(\tau_{n-1}\right)$ to cover $\operatorname{graph}(\tau)$. Hence an upper bound for the number of cubes covering $\operatorname{graph}(\tau)$ is

$$
\frac{\operatorname{var}\left(\tau_{n-1}\right)+3}{2^{-n}}=\frac{2 \sqrt{n}\left(1+O\left(\frac{1}{n}\right)\right)+3}{2^{-n}}
$$

and if we choose $h(s)=s /\left(\log \frac{1}{s}\right)^{1 / 2}$ we get the following upper bound for the box sum with respect to $h(s)$ :

$$
\begin{aligned}
& \frac{2 \sqrt{n}\left(1+O\left(\frac{1}{n}\right)\right)+3}{2^{-n}} \times \frac{2^{-n}}{\left(\log \left(1 / 2^{-n}\right)\right)^{1 / 2}} \\
& =\frac{2}{(\log 2)^{1 / 2}}\left(1+O\left(\frac{1}{n}\right)\right)+\frac{3}{2 \sqrt{n}}<\frac{2}{(\log 2)^{1 / 2}}+O\left(\frac{1}{\sqrt{n}}\right)
\end{aligned}
$$

for $n$ big enough. A lower bound is achieved in a similar way. Cover $\operatorname{graph}(\tau)$ with dyadic cubes of sidelength $2^{-n}$. Denote, as before, the number which intersect $\operatorname{graph}(\tau)$ by $\mathscr{N}\left(\tau, 2^{-n}\right)$. Since $\left|\tau_{n-1}-\tau\right|<2^{-n}$, then it is possible 
to cover $\operatorname{graph}\left(\tau_{n-1}\right)$ by $2 / 2^{-n}+\mathcal{N}\left(\tau, 2^{-n}\right)$ cubes. But a lower bound for the number of cubes which are needed to cover $\operatorname{graph}\left(\tau_{n-1}\right)$ is $\operatorname{var}\left(\tau_{n-1}\right) / 2^{-n}$. Hence $\operatorname{var}\left(\tau_{n-1}\right) / 2^{-n}-2 / 2^{-n}<N\left(\tau, 2^{-n}\right)$, and a lower bound for the lower box sum limit with respect to $h(s)$ is

$$
\frac{\left.2 \sqrt{n}\left(1-\left|O\left(\frac{1}{n}\right)\right|\right)-2\right)}{2^{-n}} \times \frac{2^{-n}}{\left(\log \left(1 / 2^{-n}\right)\right)^{1 / 2}}=\frac{2}{(\log 2)^{1 / 2}}-\left|O\left(\frac{1}{\sqrt{n}}\right)\right|,
$$

for $n$ large enough. It follows that the box sum limit with respect to $t /\left(\log \frac{1}{t}\right)^{1 / 2}$ exists and the proof is complete.

A function in $\mathscr{T}$, which distinguishes itself by its properties, and which we will call the $\lambda$-function, is $\lambda(t)=\tau\left(\frac{2}{3}\right)$.

Corollary 2.7. The Takaga function and the $\lambda$-function have logarithmic Hausdorff dimension at most $\frac{1}{2}$.

Proof. $T(t)=\tau(1)$ and $\lambda(t)=\tau\left(\frac{2}{3}\right)$.

The next theorem shows that in some sense the order of smoothness of the functions in $\mathscr{T}$ attains its maximum on the $\lambda$-function and its minimum on the Takaga function.

Theorem 2.8. $\Delta_{h}^{2} \lambda(t)=O(h)$, but $\Delta_{h}^{2} T(t)=O\left(h \log \frac{1}{h}\right)$ is best possible.

Proof. We will first prove $\lambda \in \Lambda^{1}([0,1])$. By definition this means that there is a constant $c$ so that

$$
|\lambda(t)| \leq c, \quad\left|\Delta_{h}^{2} \lambda(t)\right| \leq c h, \quad h \in\left(0, h_{0}\right],
$$

for some positive constant $h_{0}$. The first inequality in (2.11) is immediate from the construction. Choose for instance some constant $c \geq 1$. The second inequality in (2.11) is proved in the following way. Let $h>0$ be given and choose $n$ so that $2^{-n}<2 h \leq 2^{-n+1}$. Then

$$
\left|\Delta_{h}^{2} \lambda(t)\right| \leq\left|\Delta_{h}^{2} \sum_{i=0}^{n-1} 2^{-i} \gamma_{2 / 3}\left(2^{i} t\right)\right|+\left|\Delta_{h}^{2} \sum_{i=n}^{\infty} 2^{-i} \gamma_{2 / 3}\left(2^{i} t\right)\right|<10 h .
$$

The second difference, $\Delta_{h}^{2}$ of the finite and infinite sums above are calculated using function values at $t, t+h$, and $t+2 h$, i.e., points in an interval of smallest length $2 h$. It then follows from the construction of $\gamma_{2 / 3}$ that at most three terms in the finite sum have second differences which are nonzero. Since each $\Delta_{h}^{2}$ is at most $2 h$ it follows that the first term is less than $6 h$. The second term is estimated by a geometric series and is less than $4 h$. It is possible to improve the bound $10 \mathrm{~h}$ but this is good enough to prove $\lambda \in \Lambda^{1}$. That $\Delta_{h}^{2} T(t)=O\left(|h| \log \frac{1}{|h|}\right)$ is best possible, follows easily from (iii) in Theorem 2.3 and evaluation of $\Delta_{h}^{2} T(t)$ at $h=2^{-n-1}$ and $t=2^{-1}-2^{-n-1}$. We have

$$
\begin{aligned}
& \Delta_{2^{-n-1}}^{2} T\left(2^{-1}-2^{-n-1}\right)=\Delta_{2^{-n-1}}^{2}\left(\sum_{i=0}^{n} 2^{-i} \operatorname{dist}\left(2^{i} t\right)\right) \\
& =(2+\cdots+2) 2^{-n-1}=2(n+1) 2^{-n-1}=\frac{2}{\log 2}|h| \log \frac{1}{|h|} .
\end{aligned}
$$

Theorem 2.9. Every function $\tau_{a} \in \mathscr{T}, a \in[0,1]$, is nowhere differentiable.

Proof. The continuity of $\tau_{a}$ is a consequence of the definition of $\tau_{a}$ as uniform limits of continuous functions. To show the nowhere differentiability, notice 
that, if $\tau_{a}$ were differentiable at the point $x$, then we would have that

$$
\lim _{x \in\left(x_{1}, x_{2}\right)} \frac{\tau_{a}\left(x_{1}\right)-\tau_{a}\left(x_{2}\right)}{x_{1}-x_{2}} \text { as }\left|x_{1}-x_{2}\right| \rightarrow 0
$$

exists and is finite. For every $x \in[0,1]$ we have $\{x\}=\bigcap_{\nu=1}^{\infty} I_{\nu}$ for a sequence of nested and closed dyadic intervals such that length $\left(I_{\nu}\right)=2 \times \operatorname{length}\left(I_{\nu+1}\right)$. The incremental ratios in (2.12) corresponding to the endpoint values of $I_{\nu}$ and $I_{\nu+1}$, respectively, differ by construction by exactly \pm 1 for every $\nu$. Hence the limits in (2.12) cannot exist and the functions are nowhere differentiable.

\section{ACKNOWLEDGMENT}

The authors thank Professor Roland Häggkvist, Umeå University, for suggesting a combinatorial identity which made part of the proof of Theorem 2.3 shorter.

The second author wishes to thank the School of Mathematics of Georgia Institute of Technology, which he was visiting during 1990-1991, for its support.

\section{REFERENCES}

[BIN] O. V. Besov, V. P. Il'in, and S. M. Nikol'skii, Integral representations of functions and imbedding theorems, Wiley, New York, 1978.

[FAL1] K. J. Falconer, The geometry of fractal sets, Cambridge Tracts in Math., vol. 85, Cambridge Univ. Press, Cambridge and New York, 1985.

[FAL2] _ Fractal geometry, Mathematical Foundations and Applications, Wiley, New York, 1990.

[HAG] R. Häggvist, Personal communication.

[HAT] M. Hata, Topological aspects of self-similar sets and singular functions, Fractal Geometry and Analysis (J. Belair and S. Dubuc, eds.), Kluwer, Dordrecht, 1991.

[HOB] E. W. Hobson, The theory of functions of a real variable, Dover, New York, 1957.

[JWL] A. Jonsson and H. Wallin, Function spaces on subsets of $\mathbb{R}^{n}$, Math. Rep., Harwood Acad. Publ., London, 1984.

[KAP] J. L. Kaplan, J. Mallet-Paret, and J. A. Yorke, The Lyapunov dimension of a nowhere differentiable attracting torus, Ergodic Theory Dynamical Systems 4 (1984), 261-281.

[KNP] K. Knopp, Ein einfaches Verfahren zur Bildung stetiger nirgends differenzierbarer Funktionen, Math. Z. 2 (1918), 1-26.

[MAL] R. D. Mauldin and S. C. Williams, On the Hausdorff dimension of some graphs, Trans. Amer. Math. Soc. 298 (1986), 793-803.

[MAN] B. B. Mandelbrot, Fractals: form, chance and dimension, Freeman, San Francisco, CA, 1977.

[RHA] G. de Rham, Sur un example de fonction continue sans dérivée, Enseign. Math. 3 (1957), 71-72.

[ROG] C. A. Rogers, Hausdorff measure, Cambridge Univ. Press, London and New York, 1970.

[STE] E. M. Stein, Singular integrals and differentiability properties of functions, Princeton Univ. Press, Princeton, NJ, 1970.

[TAK] T. Takaga, A simple example of a continuous function without derivative, Proc. Phys. Math. Soc. Japan 1 (1903), 176-177.

[WAE] B. L. van der Waerden, Ein einfaches Beispiel einer nicht-differenzierbaren stetigen Funktion, Math. Z. 32 (1930), 474-475. 
[WEI] K. Weierstrass, Mathematische Werke, Mayer and Müller, Berlin, 1894-1927.

[WIN] P. Wingren, Imbeddings and characterizations of Lipschitz spaces on closed sets, Department of Mathematics, University of Umeả 5 (1985).

[ZYG] A. Zygmund, Trigonometric series, 2nd ed., Vols. I and II, Cambridge Univ. Press, 1959.

School of Mathematics, Georgia Institute of Technology, Atlanta, Georgia 30332

E-mail address: deliu@math.gatech.edu

Department of Mathematics, University OF UMeȦ, UmeẢ, S-901 87 SWeden

E-mail address: wingren@biovax.umdc.umu.se 